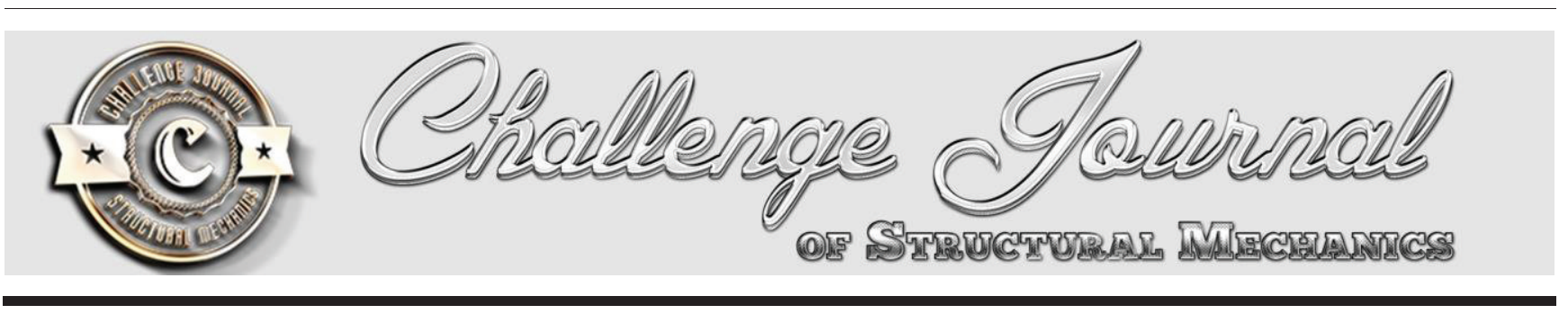

\title{
Assessment of bonding defects in FRP reinforced structures via ultrasonic technique
}

\author{
Emma La Malfa Ribolla, Mohsen Rezaee Hajidehi, Giuseppe Fileccia Scimemi, \\ Antonino Spada, Giuseppe Giambanco* \\ Department of Civil, Environmental, Aerospace, Materials Engineering, University of Palermo, 90128 Palermo, Italy
}

\begin{abstract}
ABS T RACT
Fiber reinforced polymer (FRP) composite systems are widely used for the rehabilitation of concrete structures such as building that need to resist to seismic loads, bridges that have to carry heavier traffic loads. The technique consists in bonding the composite plate to the concrete surface element in order to increase the flexural capacity. A proper attachment of the FRP plate to the concrete surface is necessary for the efficiency of the load transfer between the reinforcement and the substrate. In this work, the quality of composite bonding is characterized through ultrasonic testing. The proposed technique is relative to a time domain analysis of the ultrasonic signals and couples the Akaike Information Criterion (AIC), used as automatic onset signal detection, and the Equivalent Time-Length (ETL), used as an indicator of the quantity of energy propagating through the bonding. It has been tested both numerically and experimentally, in vitro, using samples with imposed well- known defects.
\end{abstract}

\section{ART I CLE I N F O}

Article history:

Received 4 July 2016

Accepted 26 August 2016

\section{Keywords:}

FRP

Bonding

Nondestructive testing

Ultrasonic waves

\section{Introduction}

Over the past decades, fiber reinforced polymer (FRP) composite systems have been used for the retrofit of concrete structures. The most common FRP rehabilitation system consists of laminates of carbon- or glass- reinforced polymers (CFRP or GFRP), bonded to the surface of the concrete by means of epoxy adhesive. This technology offers exclusive advantages with respect to the traditional strengthening techniques, e.g. immunity to corrosion, low weight and excellent mechanical properties. The efficiency of a FRP rehabilitation system is highly influenced by its integrity. In particular, the quality of the FRP-concrete interfacial bonding is a critical parameter affecting the structural performance and life expectancy of retrofitted structures since it governs the stress transfer from the concrete to the FRP composite. During the installation of the composite system, improper cure can cause voids, inclusions, debonds and delaminations at the FRP-substrate level. The effects of the common defects are discussed by Kaiser and Karbhari (2003). In addition to this aspect, one of the main problem related to the use of FRP strengthening is the detachment phenomenon, which consists in the sudden and brittle decohesion of the FRP reinforcement from the quasi-brittle support element (Toti et al., 2013; Cottone and Giambanco, 2009).

To evaluate the FRP-substrate defects, non-destructive techniques (NDT) can be used. For this specific application, diversified NDT methods have been advanced and an extensive literature review was carried out by Dong and Ansari (2011) and Kaiser and Karbhari (2004a; 2004b). Infrared thermography has been used to monitor FRP strengthened reinforced concrete bridge columns (Jackson et al., 2000), bridge decks (Halabe et al., 2007), and reinforced concrete beams (Shih et al., 2003). Microwave testing has been used to identify artificially induced disbonds and delaminations within the FRP and at the FRP-to-concrete interface (Akuthota et el., 2004), and delaminations in concrete beams fabricated and strengthened with CFRP (Ekenel et al., 2004). Mirmiran et al. (1999) investigated the applicability of acoustic emission (AE) technology to inspect hybrid CFRP tubes filled with concrete and to correlate 
the acoustic emission parameters to the state of stress in concrete. Degala et al. (2009) describe an AE approach to monitor the failure mechanism in reinforced concrete slabs retrofitted with CFRP strips.

In accordance with the combined considerations of defect detectability and practicality of use advanced by Kaiser and Karbhari (2004b), ultrasonic tests are still classified as primary methods of choice due to the existing considerable background and wide range of instrumentation.

Many researchers performed experimental studies using ultrasonic waves for the damage detection on composite laminates (Su et al., 2006; Lestari and Qiao, 2005; Kessler et al., 2002; Alleyne and Cawley, 1992). All these studies are based on the propagation of guided ultrasonic waves (GUWs). Different types of guided waves exist depending upon the geometry of the waveguide, for instance in plate-like structures Lamb waves propagate. These waves can travel over a long distance even in materials with a high attenuation ratio. Unfortunately, however, Lamb wave testing is complicated by the existence of at least two dispersive modes at any given frequency.

Even though an extensive research exists in the framework of damage detection on composite laminates by means of GUWs propagation, less information exists about the wave propagation in FRP laminates bonded to roughly inhomogeneous materials, such as concrete. In this case, a high scattering attenuation occurs due to the presence of heterogeneities. The concrete behaves almost like a perfect absorber generating a considerable number of short-spaced echo peaks that make the defect echo not observable. Previous efforts in the ultrasonic evaluation of bonding defects at the FRP-concrete interface are represented by the work of Bastianini et al. (2001), which involves longitudinal waves propagation in CFRP and GFRP composite materials applied to different substrates (concrete, masonry, and polyurethane) and is based on the relative amplitude of the first echo peak. Kundu et al. (1999) used both Lamb wave scanning and longitudinal wave scanning, in particular pulse-echo and tone-burst C-scan. Mahmoud et al. (2010) studied the influence of aging condition of CFRP-concrete specimens on ultrasonic features, i.e. velocity, peak amplitude, average power, maximum power-frequency ratio and frequency at maximum ratio. Mirmiran and Wei (2001) used ultrasonic pulse velocity (UPV) testing to quantify the extent and progression of damage in concrete filled FRP-tubes.

In this paper, an experimental study with the aim of assessing the quality of the FRP-concrete bonding by means of ultrasonic testing is presented. The main novelty of the paper is the introduction of a statistical parameter called Equivalent Time-Length (ETL) to predict the quality of the bonding. The proposed procedure involves also the automatic determination of the onset of the signal which is performed by means of the Akaike Information Criterion (AIC) (Akaike, 1974). To interpret the experimental findings, Finite Element (FE) analysis were performed.

\section{ETL for Defect Detection}

In the case of perfect bonding between FRP and concrete, the acoustical impedance mismatch between the two media is small. In this case, the sound speed measured in the FRP and the substrate is of the same order of magnitude, therefore the energy of vibration is almost entirely transmitted to the concrete where it is largely absorbed by scattering. On the contrary, when the bond between FRP and concrete is compromised by the presence of a thin air gap, the acoustical mismatch is much bigger and the sound speed in the FRP is about 10 times bigger than the one in the air. In this condition, a higher amount of energy mostly propagates in the direction of the fibers (Fig. 1).

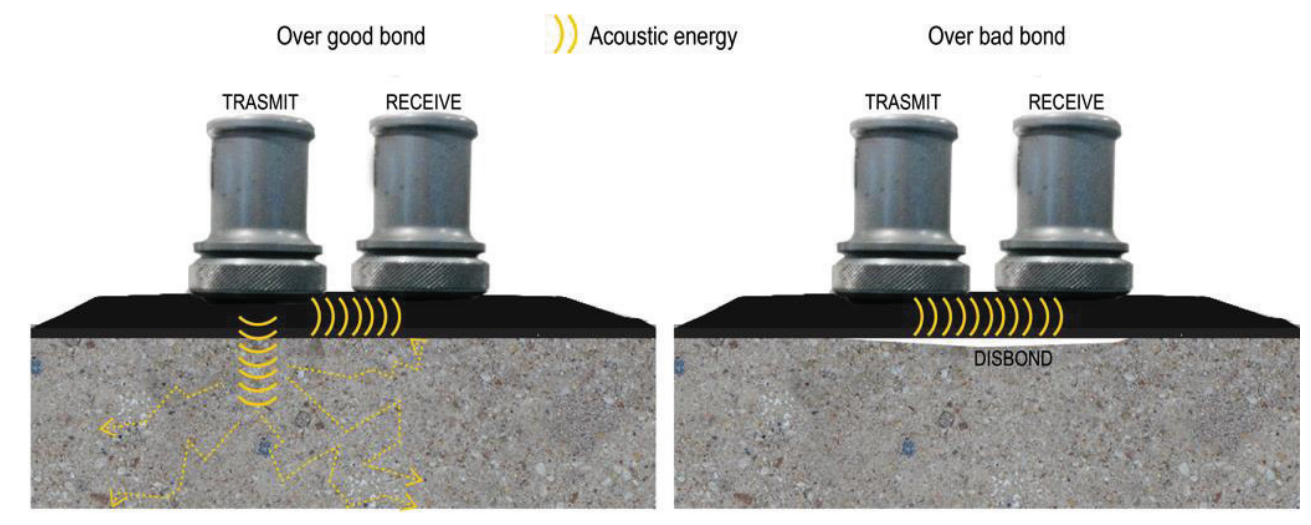

Fig. 1. Bond testing pitch-catch mode.

To quantify the aforementioned energetic phenomenon, the most common practices involve the use of the first echo amplitude (Bastianini et al., 2001), the peakto-peak or the average amplitude of the signal in a given time window (Kundu et al., 1999). However, by applying a direct-contact technique, the ultrasonic response in terms of pulses amplitude is affected by two main factors, i.e. the thin film of couplant between the probe and the medium and the pressure of the transducers on the sample. Furthermore the presence of fibers strongly affects the ultrasonic response.

The proposed approach considers the onset time of the signal, which is calculated by applying the automatic onset determination algorithm advanced by Akaike 
(1974) and used by other researchers (Maeda, 1985; Kurz et al., 2005). The algorithm allows the exact onset determination when signal and noise are in the same frequency range, which often occurs in presence of concrete as a travelling medium (Kurz et al., 2005). According to this approach, , a time series can be divided into locally stationary segments, each modeled as an autoregressive process. A signal including the onset and a first estimate of the onset time is needed. The intervals before and after the onset time are assumed to be two different stationary time series. For a fixed order autoregressive process, the point at which the AIC is minimized determines the separation point of the two time series (noise and signal) and therefore the onset point of the signal, $t_{\text {AIC. }}$. The AIC function is obtained through the following expression:

$A I C[k]=k \log (\operatorname{var}(A(k, 1)))+(N-k-1) \log (\operatorname{var}(A(1+k, N)))$,

where the term $A(k, 1)$ means that the variance function is only calculated from the current value of $k$, while $A(1$ $+k, N$ ) means that all samples ranging from $1+k$ to $N$ are taken. By calculating the minimum of Eq. (1), the onset time $t_{\text {AIC }}$ is obtained:

$$
\begin{aligned}
& A I C\left(k_{\min }\right)=\min A I C[k], \\
& t_{A I C}=k_{\text {min }} \Delta t,
\end{aligned}
$$

being $\Delta t$ the time interval between two sample points.

Successively, the following wave feature, named Equivalent Time Length (ETL) is calculated as:

$$
E T L=\sqrt{\frac{\sum_{k=A I C+1}^{N} A\left(t_{k}\right)^{2}\left(t_{k}-t_{A I C}\right)^{2}}{\sum_{k=A I C+1}^{N} A\left(t_{k}\right)^{2}}} k=[1,2, \ldots, N],
$$

where $N$ is the number of point samples, $A\left(t_{k}\right)$ is the amplitude of the signal at the time $t_{k}$. It should be mentioned that a similar parameter is adopted in the framework of time-domain measurements of partial discharge pulse (Contin et al., 2002). The difference with respect to the present study is that in Eq. (4) $t_{\mathrm{AIC}}$ is named $t_{0}$ and is defined as the time-barycenter of the energy of the signal. In the framework of partial discharge pulses, the ETL, together with the equivalent bandwidth, provide an intuitive and compact representation of the detected signals. From Eq. (4), it is noted that the ETL returns the time associated to the sum of the products of the instantaneous energies and their respective square occurring time, with respect to the onset of the signal.

\section{Ultrasonic Test}

\subsection{Numerical setup}

A two dimensional FEM model was developed to simulate the ultrasonic wave propagation within a composite laminate partially or fully bonded to a concrete sample. The numerical setup follows the work of Ghose et al. (2011) where ultrasonic guided wave propagation is simulated in a multi-layered composite structure. The model is presented in Fig. 2(a) and consists of a $150 \times 250 \mathrm{~mm}$ concrete sample cross-section covered with a layer of CFRP, $1.4 \mathrm{~mm}$ thick. The FRP-concrete interface was modeled according to four different conditions. The ND0 model corresponds to the fully bonded condition, where the nodes in pair on the same interface are rigidly linked together. In the ND1, ND2, ND3 models, the bonding defects are introduced demerging the interfacial nodes. 4517 triangular elements were used to mesh the domain of the CFRP and concrete (Fig. 2(b)). The material properties of the CFRP and concrete materials used in the model are mentioned in Table 1 . The properties of the CFRP were defined considering the material as orthotropic with unidirectional fibers laid along the $x$-direction and the rule of mixtures (Voigt, 1889; Reuss, 1929).

Table 1. Material properties of FRP and concrete.

\begin{tabular}{cccc}
\hline Parameters & $\begin{array}{c}\text { Young's modulus } \\
{[\mathrm{GPa}]}\end{array}$ & $\begin{array}{c}\text { Density } \\
{\left[\mathrm{kgm}^{-3}\right]}\end{array}$ & Poisson's ratio \\
\hline CFRP & $\begin{array}{c}E_{11}=200 \\
E_{22}=E_{33}=12\end{array}$ & 1065 & $\begin{array}{c}v_{12}=v_{13}=0.31 \\
v_{23}=0.45\end{array}$ \\
Concrete & $E=25$ & 2300 & $v=0.33$ \\
\hline
\end{tabular}

The excitation signal was chosen by considering five cycles of sine function at $500 \mathrm{kHz}$ generated by the pulser-receiver and transmitted/received by means of the ultrasonic probes placed in contact with each others. This expedient was chosen to better simulate the experimental excitation input given to the structure. The disturbance was applied to a $2.5 \mathrm{~mm}$ long line of the CFRP boundary to simulate the excitation transmitted by the probe on the sample with displacement along the $z$-direction. To model the two dimensional geometry the case of plain strain was considered. Time dependent analysis, Lagrange quadratic type of element were used for the solution. The duration of time span for the solution was chosen to be $160 \mu$ s in such a way that the complete signal was captured at the point of observation, which is the midspan of the output line in Fig. 2(a). The time step chosen in the model was 0.01 $\mu \mathrm{s}$. 
(a)

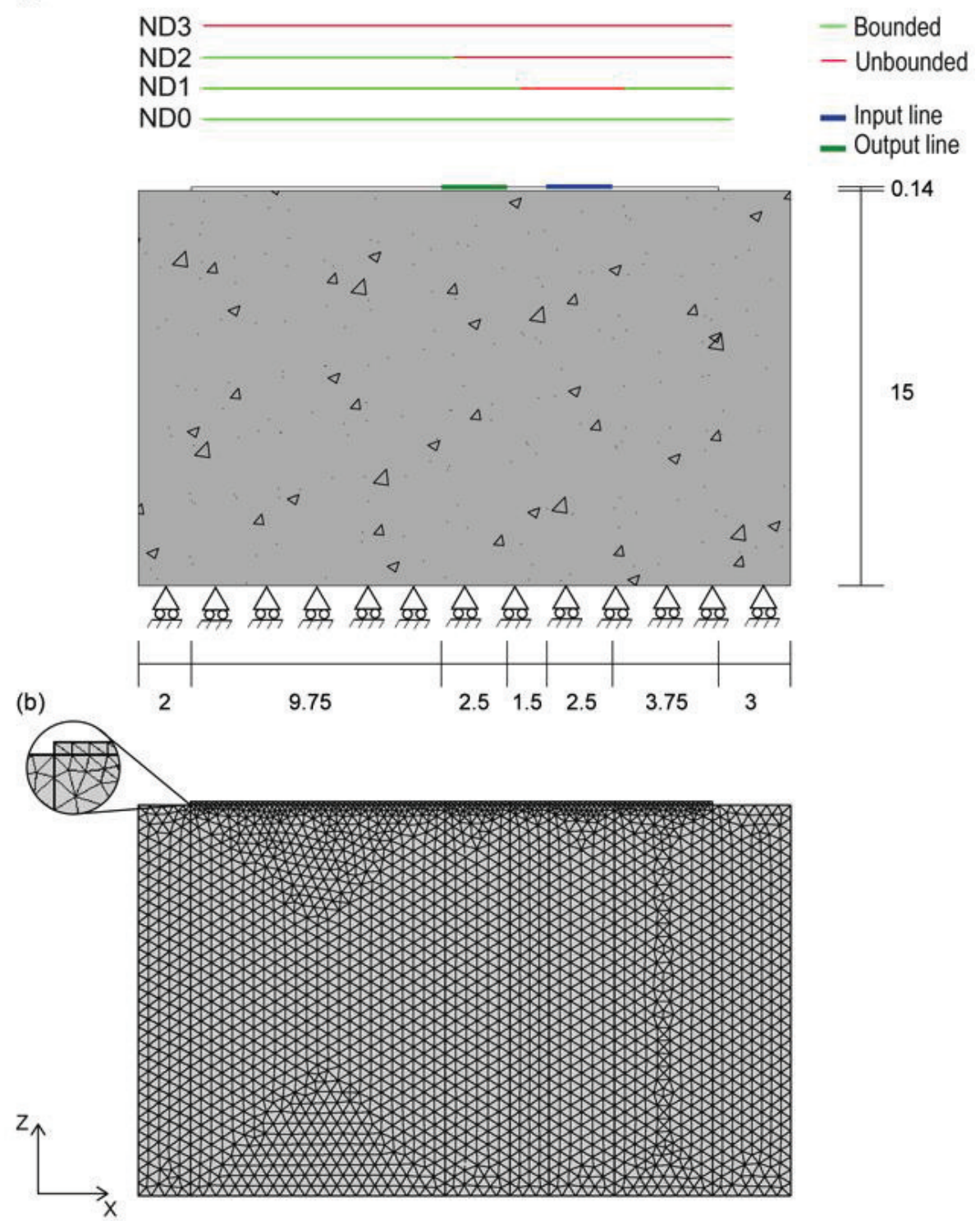

Fig. 2. Numerical simulation setup: (a) Scheme; (b) Mesh of the model. (Geometrical dimensions are expressed in centimeters.)

\subsection{FRP-concrete samples preparation}

Four concrete prisms with dimensions of $180 \times 250 \times 150 \mathrm{~mm}$ were prepared using Type I Portland Cement, water, river sand and \#67 limestone coarse aggregate as substrates. The average 28-days standard cubic strength was $30 \mathrm{MPa}$. The concrete was sandblasted to achieve a surface profile of \#4, according to the International Concrete Repair Institute (Guideline 310.1R$2008,2009)$. Bonding the FRP was executed in accordance with the manufacturer's application guideline using commercial epoxy resin. In order to simulate the lack of bonding of the FRP, three types of defects of known dimensions were imposed on three of the four samples using the interposition of different foils of Teflon between the concrete surface and the adhesive layer. The four samples were named from D0 (absence of defect, i.e. pristine state) to D3 (large defect), according to the size of the included Teflon foil. Fig. 3 shows the top view of the concrete samples at the time of the position of the defects. After mixing the two-component adhesive, this was applied using a paint brush. While the epoxy resin was still wet, dry uniaxial fabric was placed onto the substrate and pressed by means of hand pressure in the fiber direction. A second layer of epoxy was applied using a paint brush, rolling from the middle of the sheet to push out all air bubbles, ensuring that the fabric was completely saturated. The FRP was allowed to cure for 7 days at $23^{\circ} \mathrm{C}$.

\subsection{Experimental setup}

The setup shown in Fig. 4 was employed for the ultrasonic testing in the FRP- concrete samples. A housebuilt pulser-receiver was used for generating and acquiring the ultrasonic waves. In this test, five cycles of sine wave with frequency $600 \mathrm{kHz}$, were generated and employed as incident wave. Two ultrasonic transducers with frequency of 1 and $0.5 \mathrm{MHz}$ (P25 Piezo, IMG 
Ultrasuoni s.r.l.) were used in a pitch-catch mode as a transmitter and receiver transducer, respectively. The diameter of the transducer's contact surface was 25 $\mathrm{mm}$. The transducers were placed perpendicularly to the plane of the FRP reinforcement to produce a normaltype ultrasonic wave. As conducting medium, coupling gel was placed between the transducers and the specimen. The received signals were amplified and filtered by an ultrasonic preamplifier (Olympus NDT Inc., Waltham, MA, USA). The acquisition process was controlled by a house-built software Pulser 2.00. In order to obtain a more accurate time waveforms classical assumption of noise equal to zero is used, hence 50 waveforms were registered at each measurement and the meanvalue of these acquisitions was used as a reference waveform.
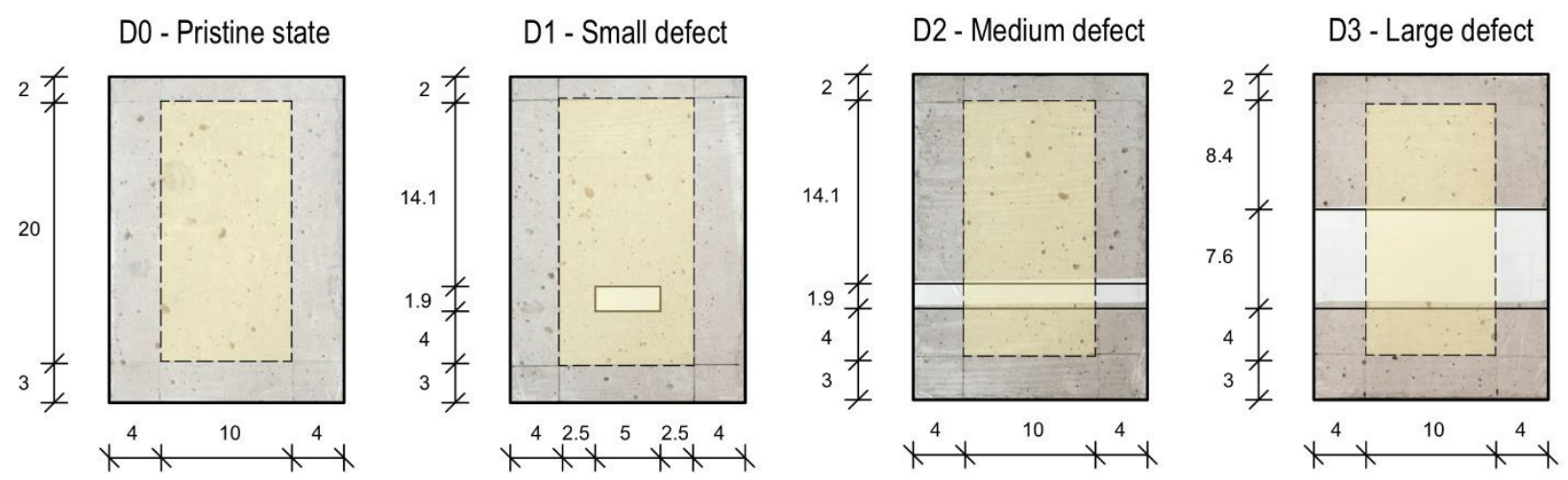

FRP bonded area

Fig. 3. Concrete samples with the Teflon foils at the time of the FRP reinforcement laying.

(Geometrical dimensions are expressed in centimeters.)
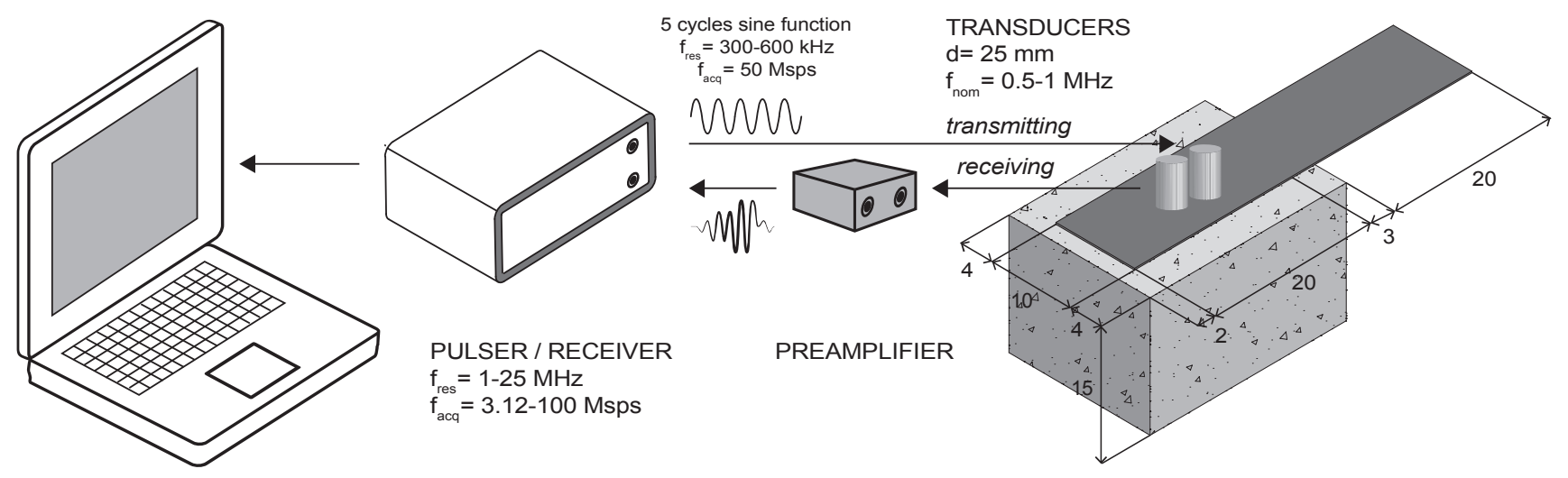

Fig. 4. Schematic configuration for the FRP-concrete ultrasonic test setup.

(Geometrical dimensions are expressed in centimeters.)

\section{Results and Discussion}

In this section, both the numerical and the experimental results are presented.

Fig. 5 shows the vertical displacement map at two different instant, equal to $6.68 \mu$ s and $15.04 \mu \mathrm{s}$. The two sub-figures are relative to ND0 model while the bottom two sub-figures are relative to the ND3 model. For the sake of shortness, only the two extreme cases are reported. The displacement maps perfectly replicate the physical phenomenon schematized in Fig. 1. In the case of perfect bonding, the waves propagates through the bulk material, conversely in the case of interfacial mismatch the waves propagates through the FRP thickness, being in this way assimilated to surface or guided waves. It should be noted that the media are numerically homogeneous, hence the scattering and the damping caused by the heterogeneities of concrete is not numerically quantifiable. In the real case, it would therefore be necessary to quantify the amount of acoustic energy which penetrate in the bulk material and the amount that is reflected at the FRP-concrete interface. Nevertheless, this requirement may be time-expensive and inconvenient.

To see how the whole energy reflection could be characterized, we present in Figs. 6(a) and (b) the waterfall chart containing the numerical and experimental waves profiles, respectively, measured at each different defect size and relative to the time interval $0-40 \mu \mathrm{s}$. The values were normalized with respect to the maximum value since the numerical pulse describes a displacement, while the experimental pulse refers to a voltage. The amplitude and the arrival of the signal change slightly, whereas the energy distribution is visibly shifted by the increase of the defect size. The variations observed were 
quantified by extracting the ETL from the time series. Together with the ETL, the Root Mean Square Deviation (RMSD) and the maximum amplitude $\left(A_{\max }\right)$ were calculated. Their numerical and experimental trends are presented in Figs. 6(c) and 6(d). The values were normalized with respect to the case of worst bonding. The latter is represented by the ND3 model for the numerical case, while, for the experimental case, this is represented by a single laminate of FRP, considered free, i.e. unbounded to concrete.
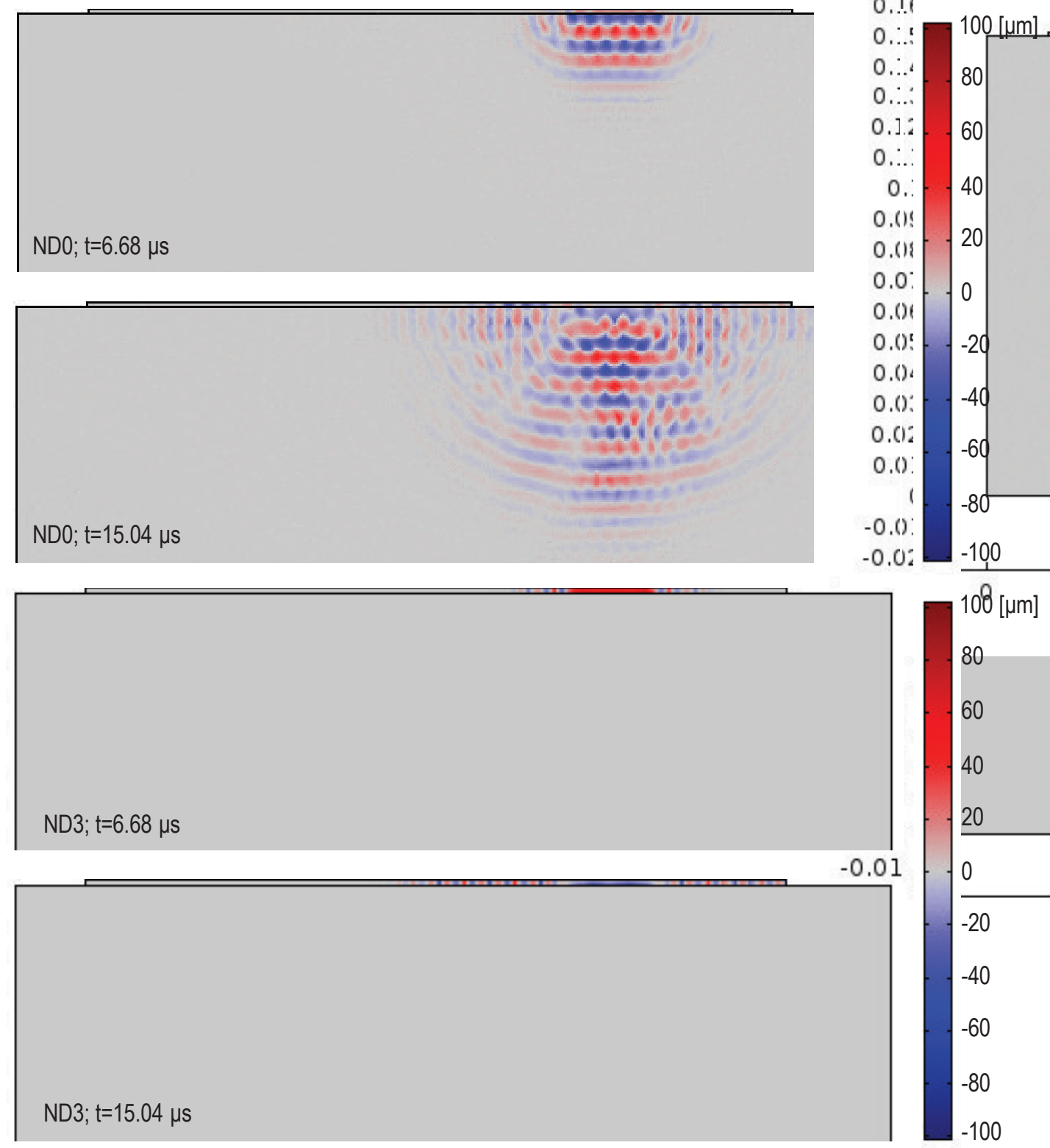

Fig. 5. Numerical results: displacement field, vertical component, relative to ND0 and ND3 model at the time $6.68 \mu$ s and $15.04 \mu \mathrm{s}$.

The results show that the statistical parameters ETL and RMSD behave monotonically. Both numerically and experimentally, the ETL is increasing with similar variation, suggesting the quantity of acoustic energy that passes through the bonding. A decreasing trend is instead observed for the RMSD, which depends on the choice of the baseline signal, that is the case of pristine state. Lastly, the maximum amplitude does not seem to have the same reliability to predict the quality of bonding. Specifically, in the case of direct active ND measurement the amplitude could be affected by external factors discussed in Section 2.
The theory of acoustic propagation in materials shows that the parameters of the backscattered ultrasonic signal depend on the ultrasonic signal frequency, reason why the ETL was in this paper calculated by considering actuation frequencies in the range 200-600 $\mathrm{kHz}$ (Fig. 7). It was found that frequencies lower than $400 \mathrm{kHz}$ are not reliable for the application developed, as visible from the linear trends associated to the ETL with not justifiable attitudes. It is however well known that lower frequencies are associated to wider wavelengths, which can be over-size with respect to the defect dimensions. 
(a)

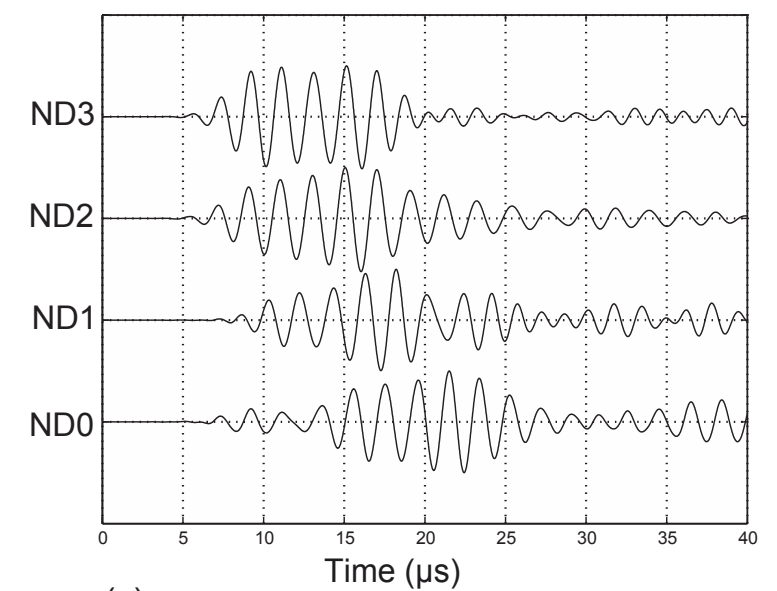

(c)

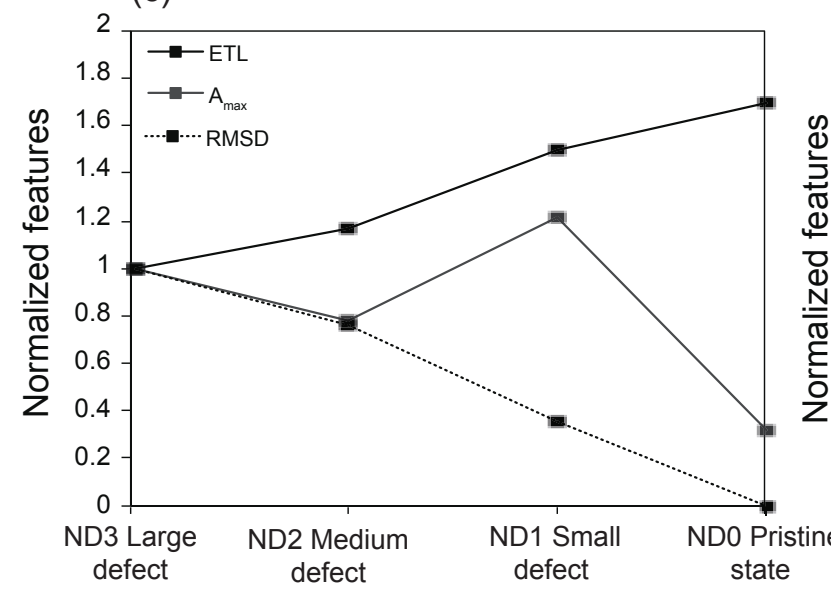

(b)

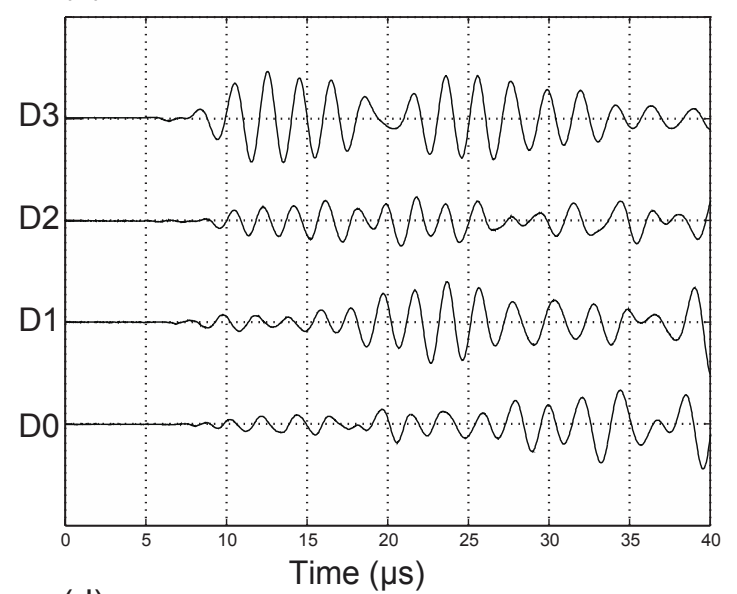

(d)

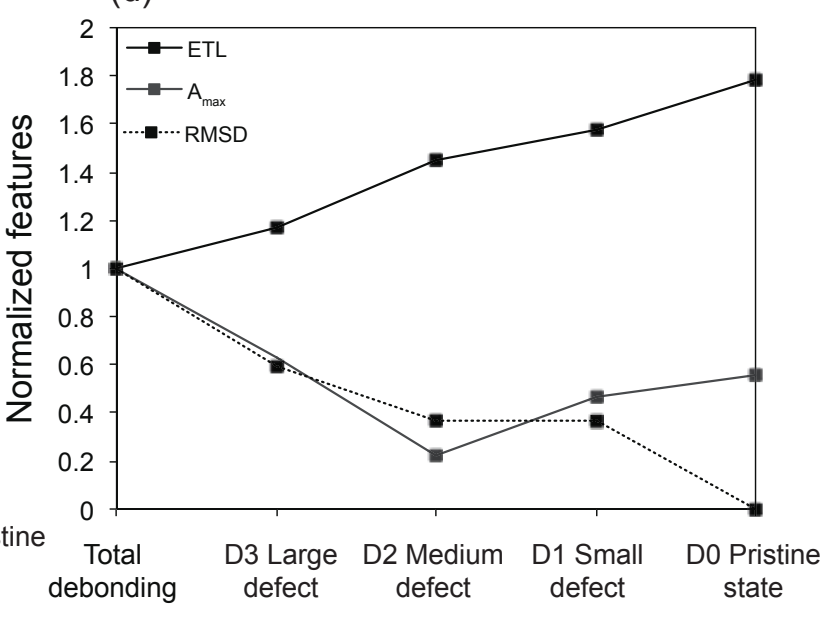

Fig. 6. FRP-concrete interface defect detection results:

(a) $z$-direction displacement profiles measured in the midspan of the output line of the numerical models; (b) $A$-scan experimental waves profiles;

(c) ETL, Maximum amplitude, RMSD calculated for the numerical;

(d) Experimental waves profiles. The values presented were normalized to the worst case of bonding.

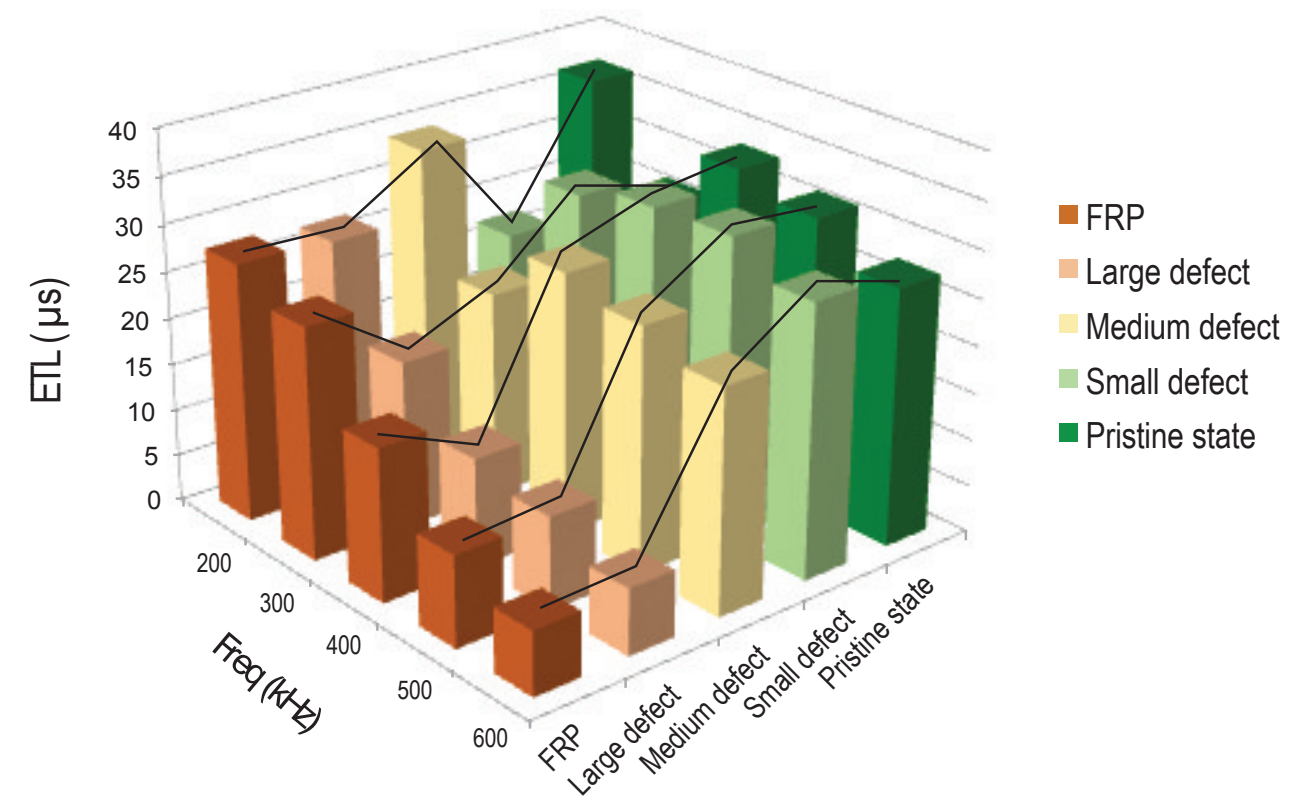

Fig. 7. FRP-concrete interface defect detection results:

ETL as a function of the quality of bonding considering different input frequencies. 


\section{Conclusions}

The integrity and the reliability of bonds are critical to the quality of FRP reinforcement in concrete structures. In this paper a pitch-catch energetic method is used as inspector method of bonding condition. As a common pitch-catch investigation, the method employs two probes, one transmitting a burst of acoustic energy into the test part and another one catching the sound propagating across the test piece. The bond condition is usually determined by considering the difference in the amplitude and/or phase between good and bad bonds. In this paper we introduce the Equivalent Time Length (ETL) as an energy distribution indicator sensitive to the quality of bonding. The inspection should be done at a fixed frequency and considering a certain time-window. Generally we observed that a bonded condition correspond to higher values of ETL since the acoustic energy is transmitted through the concrete. A disbond condition, conversely, results in lower values of ETL since the waves travel between the transmitting and receiving transducer.

We conducted a numerical analysis and laboratory experiments aimed at validating the above research hypotheses. A 2D finite element model of a FRP laminate bonded to a concrete sample was created. Different bonding conditions were modeled by decoupling the FRP-concrete interfacial nodes. Experimentally, FRP laminate with a specific bonding defects were placed into four concrete samples. The results demonstrated that the ETL increase with increasing bonding quality. The experimental results were also interpreted by comparing them to the numerical ones. We found that most of the experimental and numerical results are in good agreement each other.

\section{REFERENCES}

Akaike H (1974). Markovian representation of stochastic processes and its application to the analysis of autoregressive moving average processes. Annals of the Institute of Statistical Mathematics, 26(1), 363-387.

Akuthota B, Hughes D, Zoughi R, Myers J, Nanni A (2004). Near-field microwave detection of disbond in carbon fiber reinforced polymer composites used for strengthening cement-based structures and disbond repair verification. Journal of Materials in Civil Engineering, 16(6), 540-546.

Alleyne DN, Cawley P (1992). The interaction of Lamb waves with defects. IEEE Transactions on Ultrasonics, Ferroelectrics and Frequency Control, 39(3), 381-397.

Bastianini F, Di Tommaso A, Pascale G (2001). Ultrasonic non-destructive assessment of bonding defects in composite structural strengthenings. Composite Structures, 53(4), 463-467.

Contin A, Cavallini A, Montanari GC, Pasini G, Puletti F (2002). Digital detection and fuzzy classification of partial discharge signals. IEEE Transaction on Dielectrics and Electrical Insulation, 9(3), 335-348.

Cottone A, Giambanco G (2009). Minimum bond length and size effects in FRP-substrate bonded joints. Engineering Fracture Mechanics, 76, 1957-1976.

Degala S, Rizzo P, Ramanathan K, Harries KA (2009). Acoustic emission monitoring of CFRP reinforced concrete slabs. Construction and Building Materials, 23(5), 2016-2026.

Dong Y, Ansari F (2011). Non-destructive testing and evaluation (NDT/NDE) of civil structures rehabilitated using fiber reinforced polymer (FRP) composites. Service Life Estimation and Extension of Civil Engineering Structures, 193-222.
Ekenel M, Stephan V, Myers JJ, Zoughi R (2004). Microwave NDE of reinforced concrete beams strengthened with CFRP laminates containing surface defects and tested under cyclic loading. $16^{\text {th }}$ World Conference on Nondestructive Testing, Montreal, Canada, AugustSeptember, 2004.

Ghose B, Balasubramaniam K, Krishnamurthy CV, Rao AS (2011). Comsol based 2D FEM model for ultrasonic guided wave propagation in symmetrically delaminated unidirectional multi-layered composite structures. Proceedings of the National Seminar \& Exhibition on NonDestructive Evaluation. December 8-10, 2011.

Guideline 30.1R-2008 (2009). Guide for Surface Preparation for the Repair of Deteriorated Concrete Resulting from Reinforcing Steel Corrosion.

Halabe UB, Vasudevan A, Klinkhachorn P, GangaRao HV (2007). Detection of subsurface defects in fiber reinforced polymer composite bridge decks using digital infrared thermography. Nondestructive Testing and Evaluation, 22(2-3), 155-175.

Jackson D, Islam M, Alampalli S (2000). Feasibility of evaluating the performance of fiber reinforced plastic (FRP) wrapped reinforced concrete columns using ground penetrating RADAR (GPR) and infrared (IR) thermography techniques. Structural Materials Technology IV-An NDT Conference, 390-395.

Kaiser H, Karbhari VM (2003). Identification of potential defects in the rehabilitation of concrete structures with FRP composites. International Journal of Materials and Product Technology, 19(6), 498-520.

Kaiser H, Karbhari VM (2004a). Non-destructive testing techniques for FRP rehabilitated concrete. I: a critical review. International Journal of Materials and Product Technology, 21(5), 349-384.

Kaiser H, Karbhari VM (2004b). Non-destructive testing techniques for FRP rehabilitated concrete. I: an assessment. International Journal of Materials and Product Technology, 21(5), 385-401.

Kessler SS, Spearing SM, Soutis C (2002). Damage detection in composite materials using Lamb wave methods. Smart Materials and Structures, 11(2), 269.

Kundu T, Ehsani M, Maslov KI, Guo D (1999). C-scan and L-scan generated images of the concrete/GFRP composite interface. NDT \& E International, 32(2), 61-69.

Kurz JH, Grosse CU, Reinhardt HW (2005). Strategies for reliable automatic onset time picking of acoustic emissions and ultrasound signals in concrete. Ultrasonics, 43(7), 538-546.

Lestari W, Qiao P (2005). Application of wave propagation analysis for damage identification in composite laminated beams. Journal of Composite Materials, 39(22), 1967-1984.

Maeda N (1985). A method for reading and checking phase times in auto-processing system of seismic wave data. Zisin (=Jishin), 38(3), 365-379.

Mahmoud AM, Ammar HH, Mukdadi OM, Ray I, Imani FS, Chen A, Davalos JF (2010). Non-destructive ultrasonic evaluation of CFRP-concrete specimens subjected to accelerated aging condition. NDT \& $E$ International, 43(7), 635-641.

Mirmiran A, Shahawy M, Echary HE (1999). Acoustic emission monitoring of hybrid FRP-concrete columns. Journal of Engineering Mechanics, 125(8), 899-905.

Mirmiran A, Wei Y (2001). Damage assessment of FRP-encased concrete using ultrasonic pulse velocity. Journal of Engineering Mechanics, 127(2), 126-135.

Reuss A (1929). Berchnung der Fliegrenze von Mischkristallen auf Grund der Plastizittsbedingung fr Einkristalle. Zeitschrift fr Angewandte Mathematik und Mechanik, 9(1), 49-58 (in German).

Shih JKC, Tann DB, Hu CW, Delpak R, Andreou E (2003). Remote sensing of air blisters in concrete-FRP bond layer using IR thermography. International Journal of Materials and Product Technology, 19(1-2), 174-187.

Su Z, Ye L, Lu Y (2006). Guided Lamb waves for identification of damage in composite structures: A review. Journal of Sound and Vibration, 295(3), 753-780.

Toti J, Marfia S, Sacco E (2013). Coupled body-interface nonlocal damage model for FRP detachment. Computer Methods in Applied Mechanics and Engineering, 260, 1-23.

Voigt W (1889). Ueber die Beziehung zwischen den beiden Elasticittsconstanten isotroper Krper. Annalen der Physik, 274(12), 573-587 (in German). 